\title{
Prostatakarzinome organschonend hypofraktioniert bestrahlen
}

Die mittels Computertomografie (CT) bzw. Magnetresonanztomografie (MRT) geplante und kontrollierte Strahlentherapie (RT) hat zusammen mit entsprechender Software die Entwicklung der dreidimensionalen konformalen RT (3DCRT) und der intensitätsmodulierten RT (IMRT) ermöglicht. Nun wurde die hypofraktionierte Anwendung beider Techniken bei Prostatakrebs verglichen.

$\mathrm{R}^{\mathrm{a}}$ ndomisiert erhielten 215 Männer mit lokal begrenztem Prostatakarzinom eine hypofraktionierte Bestrahlung mit 70 Gy in 25 Fraktionen $(2,8$ Gy/d) entweder mittels IMRT $(\mathrm{n}=109)$ oder mittels 3DCRT $(n=106)$. Keiner der Patienten durfte sich zuvor einer Prostatektomie oder Chemotherapie unterzogen haben. Prospektiv erhoben die Forscher als primäre Endpunkte die akute und späte gastrointestinale und urogenitale Toxizität nach den modifizierten Kriterien der Radiation Therapy Oncology Group. Die biochemische Kontrolle wurde nach den Phoenix-Konsensuskonferenz-Kriterien ermittelt. Eine fehlende biochemische Kontrolle liegt da- nach vor, wenn der Nadir des Prostataspezifischen Antigens (PSA) um $2 \mathrm{ng} / \mathrm{ml}$ und mehr steigt.

Eine akute urogenitale Toxizität vom Grad $\geq 2$ wiesen $9 \%$ der Patienten im IMRT-und $27 \%$ im 3DCRT-Arm auf $(p=0,001)$. Akute gastrointestinale Toxizitäten derselben Schwere erlebten $7 \%$ der Patienten bei IMRT und $24 \%$ der Patienten bei 3DCRT ( $p=0,001)$. Der Vorteil für IMRT hielt auch in der weiteren Beobachtungszeit an: Späte, d. h. nach dem 6. Monat nach Therapie auftretende urogenitale Toxizitäten vom Grad 2 und höher gab es bei 3,7\% der Patienten im IMRT- und bei $12,3 \%$ im 3DCRT$\operatorname{Arm}(\mathrm{p}=0,02)$. Die entsprechenden Wer- te für späte gastrointestinale Toxizitäten lagen bei 6,4 und $21,7 \%(p=0,001)$. Die 5-Jahresrate der Freiheit von biochemischem Therapieversagen lag mit 95,4\% in der IMRT-Gruppe und $94,3 \%$ in der 3DCRT-Gruppe $(\mathrm{p}=0,678)$ in beiden Armen vergleichbar hoch.

Fazit: Die hypofraktionierte Bestrahlung ermöglichte bei beiden Verfahren eine vergleichbar gute biochemische Kontrolle des Prostatakarzinoms. Dabei ging die IMRT aber mit einer deutlichen Reduktion der frühen und späten urogenitalen und gastrointestinalen Toxizitäten vom Grad 2 und höher einher. Das bestätigt den weltweit verbreiteten Einsatz der IMRT trotz höherer Kosten als bei 3DCRT.

Friederike Klein

Viani GA et al. Intensity-modulated radiotherapy reduces toxicity with similar biochemical control compared with 3-dimensional conformal radiotherapy for prostate cancer: A randomized clinical trial. Cancer. 2016;122(13):2004-11.

\section{Überlebenserwartung bei mCRPC}

\begin{abstract}
Wie viel Lebenszeit noch bleibt, wenn ein kastrationsresistentes Prostatakarzinom gestreut hat, hängt davon ab, wo sich Metastasen gebildet haben.
\end{abstract}

$\mathrm{V}$ iszerale Absiedlungen haben sich bei Männern mit metastasiertem kastrationsresistentem Prostatakrebs (mCR$\mathrm{PC}$ ) in mehreren Studien als prognostisch besonders ungünstig erwiesen. Die Risikoabschätzungen basieren allerdings, da es sich um vergleichsweise seltene Ereignisse handelt, auf entsprechend kleinen Fallzahlen.

US-amerikanische Ärzte haben nun eine größere Datenbasis analysiert: 9 Phase-III-Studien mit zusammen 8.820 Patienten unter Docetaxel-haltiger Therapie. Ihre Zahlen bestätigen die vorausgegangenen Beobachtungen, dass Leberund Lungenmetastasen mit einer besonders hohen Letalität einhergehen. Die Patienten mit mCRPC waren zwischen
1999 und 2012 in einem medianen Alter von 68 Jahren in die Studien aufgenommen worden. Während der Nachbeobachtungszeit von median 21,8 Monaten waren insgesamt 5.470 von ihnen gestorben.

Die meisten Patienten (72,8\%) hatten unter Knochenmetastasen gelitten: Bei $42,9 \%$ der Gesamtkohorte waren ausschließlich die Knochen, bei 29,8\% auch die Lymphknoten betroffen. Viszerale Metastasen waren bei 20,8\% der Studienteilnehmer diagnostiziert worden, am häufigsten in Lungen- und Leberfiliae (bei 9,1 und 8,6\% aller Patienten). Bei den übrigen 6,4\% waren die Absiedlungen auf die Lymphknoten beschränkt.

Die kürzesten Gesamtüberlebenszeiten (OS) hatten Patienten mit Lebermetastasen. Sie lebten noch median 13,5 Monate, gefolgt von Patienten mit Lungenmetastasen mit 19,4 und Patienten mit Knochenmetastasen (mit oder ohne Lymphknotenbeteiligung) mit 21,3 Mo- naten. Patienten, bei denen ausschließlich die Lymphknoten befallen waren, hatten im Median noch 31,6 Monate zu leben.

Fazit: Wie vom Team um Susan Halabi postuliert, war das Mortalitätsrisiko von mCRPC-Patienten beim Vorliegen von Lebermetastasen um $52 \%$ höher als bei Lungenmetastasen. Letztere wiederum erhöhten die Mortalitätsrate signifikant im Vergleich zu Knochenmetastasen um $14 \%$.

Zwar spiegeln die Überlebenszeiten möglicherweise nicht die aktuelle Situation wider, weil Abirateron und Enzalutamid in den Studien noch kaum zum Einsatz kamen. Trotz dieser Einschränkung sehen Halabi und Kollegen in ihren Ergebnissen eine Orientierungshilfe sowohl für die Planung von Studien als auch für Behandlungsentscheidungen.

Beate Schumacher

Halabi S et al. Meta-Analysis Evaluating the Impact of Site of Metastasis on Overall Survival in Men With Castration-Resistant Prostate Cancer. J Clin Oncol. 2016;34(14):1652-9. 